\title{
C-MAS: The Cloud Mutual Authentication Scheme
}

\author{
Zhenpeng Liu, Fenglong Wu \\ College of Mathematics and Computer Science, Hebei \\ University, Baoding 071002, China \\ E-mail: lzp@hbu.edu.cn, 323wfl@163.com
}

\author{
Kaiyu Shang,Wenlei Chai \\ Network Center, Hebei University \\ Baoding 071002, China \\ E-mail: shang@hbu.edu.cn cwl@hbu.edu.cn
}

\begin{abstract}
A cloud mutual authentication scheme (C-MAS) is proposed to solve the problem of authentication between user and cloud computing server. Trusted computing technology and traditional smart card methods are used in cloud computing service platform. The scheme completes the authentication of both sides in cloud computing, generates the session key according consulting, at the same time, verifies the credibility of cloud service platform. Analysis shows that our scheme can resist various kinds of possible attacks, so it is therefore more secure than other schemes. And the computing time meet the requirements of cloud computing environment.
\end{abstract}

Keywords-Cloud Computing; Identity authentication; Trusted Computing; Smart Card Introduction

\section{INTRODUCTION}

As a new concept and technology, cloud computing has attract extensive attention in the world [1]. Cloud security is the most problem in cloud computing [2]. In cloud computing mode, the user's data stored in cloud service provider data center and calculation operation also worked in the environment. Users shared data center and all kinds of resources. In order to ensure the safety of the cloud system, must build a strong mutual authentication mechanism [3]. Due to the special advantages of the trusted computing technology in network, trusted computing technology is applied to cloud computing service platform. A mutual authentication scheme based on the trusted cloud computing platform is proposed to solve the problem of mutual authentication between user and cloud computing server in the cloud computing service environment.

\section{Trusted Cloud Computing Platfrom}

The core of the trusted computing technology is trusted platform module (TPM) which is embedded in the terminal platform. TPM provides hardware security for various credible mechanism and function in all types of computing platform [4].

Authentication is the important mechanism and the foundation to realize the security of cloud computing system. It provides the security guarantee for cloud user's real identity [5]. Compared with the traditional security mechanism, utilizing the TPM has more security and privacy. The TPM takes the unique encryption key into hardware which can not encroach. The root storage key which store in TPM key management bottom, for a trusted platform module owner is unique. Using the trusted platform module which is in hardware architecture, the server can create a public and private key instance: (PK, SK). This key is root storage key derivative key, and specific to hardware of server [6]. From the system start to establish execution environment, TPM measures platform hardware and software components. The corresponding hash value integrity measure information saved in a group of PCR register of TPM. At the same time the created events recorded in storage measurement log (SML). PCR value and SML value are together used to verify the platform state for user. The trusted cloud computing platform framework is shown in figure 1:

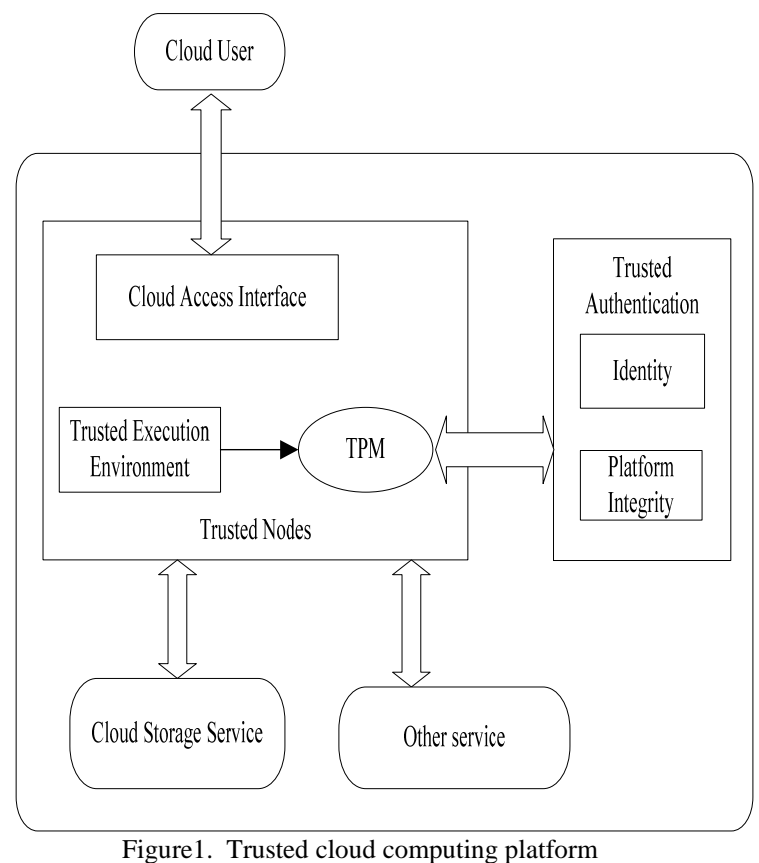

\section{Trusted Mutual Authentication Scheme}

The scheme scene model is shown in figure 2. User, cloud server (TPM) and CA (Certificate Authority) can access each other in the internet. After finishing registration in cloud server platform, the user gets smart card and accesses to cloud server through client. Cloud server and the user finished the attestation. CA manages the public key which is corresponds to the identity of cloud server (TPM). The user who has smart card can proposes query for CA, tests the consistency about public key and cloud server identity [7]. 


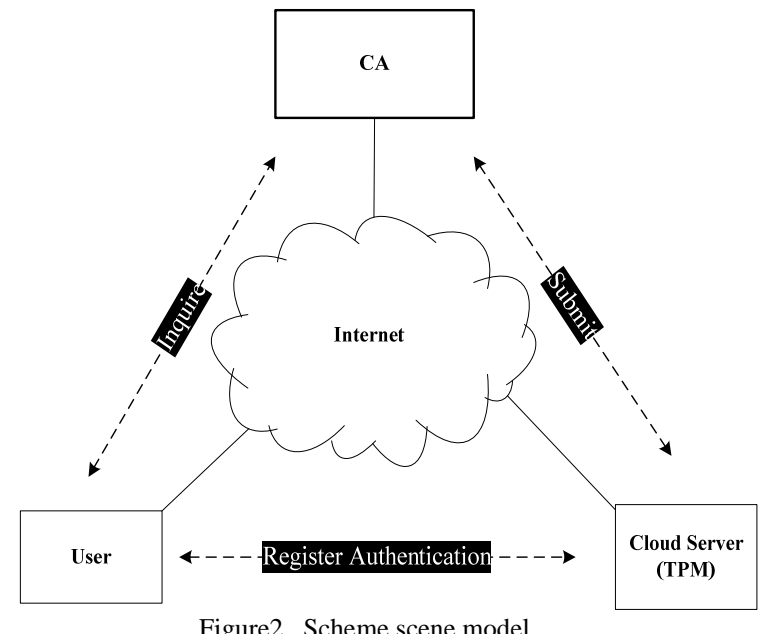

Figure2. Scheme scene model

User sends request to cloud server through the smart card. The scheme include registration, login and password change three phase in the process of implementation. We assume that the user's terminal platform is credible and in registered stage user and cloud server provider are both honest [8]. The symbols in this paper are shown in table I :

TABLE I . NOTATIONS

\begin{tabular}{|c|c|}
\hline Symbol & Definition \\
\hline S & Cloud Server \\
\hline U & User \\
\hline ID & User Identity \\
\hline PW & User Password \\
\hline $\mathrm{h}(\bullet)$ & Hash Function \\
\hline PK & Public Key \\
\hline SK & Private Key \\
\hline$\|$ & Concat \\
\hline$\oplus$ & $\mathrm{XOR}$ \\
\hline $\mathrm{T}$ & System Time Stamp \\
\hline $\operatorname{Sig}\{\}_{S K}$ & Signature \\
\hline $\log \{\}$ & Security Measure Log \\
\hline $\mathrm{K}_{\mathrm{US}}$ & Session Key \\
\hline
\end{tabular}

\section{A. Registration Phase}

The registration phase includes two parts, one is the user sends registration request to cloud server, and the second is cloud server issues registration information to the user. The steps are as follows: (1) When user sending register request to cloud server, user should choose a identity (ID), password (PW) and a random number "n", calculate $h(P W \oplus n)$, send ID and $h(P W \oplus n)$ to cloud server. (2) Cloud server calculates $\mathrm{PID}=\mathrm{h}(\mathrm{ID} \| \mathrm{x}), \mathrm{R}=\mathrm{PID} \oplus \mathrm{h}(\mathrm{PW} \oplus \mathrm{n})$ after receive user registration request. " $\mathrm{X}$ " is the secret number cloud server select, and for security reason it should be greater than 100 bit. Choose a big prime number "P" and g $\in \mathrm{GF}(\mathrm{P})$; Cloud server uses trusted platform module (TPM) which in hardware architecture to create public key and privacy key
(PK, SK); Cloud server takes the message $\{\mathrm{R} 、 \mathrm{P} 、 \mathrm{~g} 、 \mathrm{~h}$ (.)、 $\mathrm{PK}\}$ issued to user smart card through security channel. (3) User takes ID and the number "n" input to smart card, and now the information $\{I D 、 R 、 P 、 g 、 h() 、 P K 、 n$.$\} stored$ in smart card. And user doesn't need to remember the number "n".

\section{B. Login Phase}

User takes smart card into the terminal card reader, enter ID and PW. Smart card first checks whether the format of ID is valid, if the ID is invalid, the smart card refused user's authentication request. If pass, perform the following operation:

- User generates a temporary random number "r" and a secret number "a", calculates $\mathrm{PID}=\mathrm{R} \oplus \mathrm{h}(\mathrm{PW} \oplus \mathrm{n})$, $\mathrm{K}_{\mathrm{U}}=\mathrm{g}{ }^{\mathrm{a}} \bmod \mathrm{P}, \mathrm{C}_{1}=\mathrm{PID} \oplus \mathrm{h}(\mathrm{r} \oplus \mathrm{n}), \mathrm{C}_{2}=\mathrm{h}(\mathrm{h}(\mathrm{r} \oplus \mathrm{n}) \oplus$ $\mathrm{T}_{1}$ ). $\mathrm{T}_{1}$ is the local time stamp. And then user calculates $\mathrm{H}_{\mathrm{U}}=\mathrm{h}\left(\mathrm{ID}, \mathrm{C}_{1}, \mathrm{C}_{2}, \mathrm{~K}_{\mathrm{U}}, \mathrm{T}_{1}\right.$ ), sends the message $M_{1}=\left\{I D, C_{1}, C_{2}, K_{U}, T_{1}, H_{U}\right\}$ to cloud server.

- After receive the message $M_{1}$, Cloud server checks $\mathrm{T}_{1}{ }^{\prime}-\mathrm{T}_{1} \leqslant \triangle \mathrm{T} . \mathrm{T}_{1}{ }^{\prime}$ is cloud server current time stamp, $\triangle \mathrm{T}$ is the legal communication delay. If established, then calculates $\mathrm{H}_{\mathrm{U}}^{\prime}=\mathrm{h}\left(\mathrm{ID}, \mathrm{C}_{1}, \mathrm{C}_{2}, \mathrm{~K}_{\mathrm{U}}, \mathrm{T}_{1}\right)$. Through judge $\mathrm{H}_{U}$ and $\mathrm{H}_{U}$ is consistent, cloud server verify the integrity of user's message. If the verification passed, calculates $\mathrm{PID}=\mathrm{h}(\mathrm{ID} \| \mathrm{x}$ ), $\mathrm{C}_{1}{ }^{\prime}=\mathrm{PID} \oplus \mathrm{C}_{1}$ and $\mathrm{C}_{2}{ }^{\prime}=\mathrm{h}\left(\mathrm{C}_{1}{ }^{\prime} \oplus \mathrm{T}_{1}\right)$; At last if $\mathrm{C}_{2}{ }^{\prime}$ is equal to $C_{2}$, the cloud server can determine the user is legal.

- Cloud server chooses a secret number "b" and the information $\mathrm{K}_{\mathrm{U}}$, calculates $\mathrm{K}_{\mathrm{S}}=\mathrm{g}^{\mathrm{b}} \bmod \mathrm{P}, \mathrm{K}_{\mathrm{US}}=\left(\mathrm{K}_{\mathrm{U}}\right)^{\mathrm{b}}=$ $\left(\mathrm{g}^{\mathrm{a}}\right)^{\mathrm{b}}$ mod P. Through TPM chip cloud server calculates the platform integrity check value PCRS. $\mathrm{PCR}_{\mathrm{S}}=\mathrm{SHA}\left(\mathrm{PCR}_{0}\left\|\mathrm{PCR}_{1}\right\| \cdots \| \mathrm{PCR}_{\mathrm{N}}\right)$. Signing the message $C_{3}$ with private key SK: $C_{3}=\operatorname{Sig}\left\{C_{1}\right.$, $\left.\mathrm{PCR}_{\mathrm{S}}\right\}_{\mathrm{SK}}$; Loading platform security measure log: $\mathrm{L}=\mathrm{Log}(\mathrm{SML})$; Computing $\mathrm{H}_{\mathrm{S}}=\mathrm{h}\left(\mathrm{C}_{3}, \mathrm{~K}_{\mathrm{S}}, \mathrm{L}, \mathrm{T}_{2}\right)$, $\mathrm{T}_{2}$ is the time stamp $\mathrm{S}$ produce. At last, cloud server sends message $M_{2}=\left\{C_{3}, K_{S}, L, T_{2}, H_{S}\right\}$ to the user.

- After receive the news $\mathrm{M}_{2}$, user first checks the time stamp $T_{2}$. Judge $T 2 '-T 2 \leqslant \triangle T$ is right or not. $T_{2}{ }^{\prime}$ is the user current time stamp. If right, calculates $\mathrm{H}_{\mathrm{S}}{ }^{\prime}=\mathrm{h}\left(\mathrm{C}_{3}, \mathrm{~K}_{\mathrm{S}}, \mathrm{L}, \mathrm{T}_{2}\right)$, judges Hs' and $\mathrm{Hs}$ is equal or not. If equal, user decryption $\mathrm{C}_{3}$ gets the value of $\mathrm{C}_{1}$ 'and $\mathrm{PCR}_{\mathrm{S}}$. If $\mathrm{h}(\mathrm{r} \oplus \mathrm{n})$ is equal to $\mathrm{C}_{1}$ ', the user can confirm cloud server. At last, user calculates the final session key: $K_{U S}=\left(K_{S}\right)^{a} \bmod P=\left(g^{b}\right)^{a} \bmod P$.

- User verify the cloud computing service platform integrity to ensure the server system configuration information meet the safety strategy. That means judges the server status is credible or not. Recounting the $\mathrm{PCR}_{\mathrm{S}}{ }^{\prime}=\mathrm{SHA}\left(\mathrm{PCR}_{0}\left\|\mathrm{PCR}_{1}\right\| \cdots \|\right.$ $\mathrm{PCR}_{\mathrm{N}}$ ) make use of the value of $\mathrm{L}$. If $\mathrm{PCR}_{\mathrm{S}}$ ' and 
$\mathrm{PCR}_{\mathrm{S}}$ are consistent, we can confirm the integrity of cloud server platform.

Finally, the user and cloud server complete the mutual identity authentication. And at the same time user confirm the integrity of server platform. The cloud server provides services and data which is protected by session key $\mathrm{K}_{\mathrm{US}}$ to user.

\section{Password Change Phase}

After finished login and authentication phase, the user can request change password. First enter the new password $\mathrm{PW}_{\text {new }}$, the smart card calculates $\mathrm{R}_{\text {new }}=\mathrm{R} \oplus \mathrm{h}(\mathrm{PW} \oplus \mathrm{n}) \oplus$ $\mathrm{h}\left(\mathrm{PW}_{\text {new }} \oplus \mathrm{n}\right)=\mathrm{h}(\mathrm{ID} \| \mathrm{x}) \oplus \mathrm{h}\left(\mathrm{PW}_{\text {new }} \oplus \mathrm{n}\right)$. Then $\mathrm{R}_{\text {new }}$ will take the place of $\mathrm{R}$ stored in smart card.

\section{SCHEME ANALYSES}

\section{A. Security Analysis}

This paper provides a mutual authentication scheme for user and cloud service provider in cloud computing environment. With the help of TPM inherent attribute, the scheme realizes the authentication for cloud server identity and platform status. The specific situation analysis is as follows:

- Replay Attack: In login authentication stage, the user sends the message includes time stamp $T_{1}$. The time stamp can effectively prevent message replay attack.

- Denial of service attack: User can request access to cloud server by providing the correct ID. The ID has been verified from smart card. User ID and smart card are bound together. If can't receive the smart card and the corresponding ID at the same time, the attacker can't launch denial of service attack to cloud server.

- Password guessing attack: Including online password guessing attack and offline password guessing attack. According to the online password guessing attack the system can restrict user unit time login number to stop the online password guessing attack. The attacker can get the information stored in the smart card when they get the lost smart card. The information are $\{\mathrm{ID} 、 \mathrm{R} 、 \mathrm{P} 、 \mathrm{~g} 、 \mathrm{~h}(). 、 \mathrm{PK} 、 \mathrm{n}\}$, but the attack can't get the information about user's password. In addition, the message $\mathrm{h}$ (ID $\| \mathrm{x}$ ) is confidential for others. So attacker can't launch offline password guessing attack through $\mathrm{R}$ and $\mathrm{n}$.

- Impersonation attack: Assume that the attacker intercept the message of $\mathrm{M}_{1}$, obtained the value of $\mathrm{C}_{1}$ and $C_{2}$. But the attacker don't know the secret number "a" of user, the attacker will still can't camouflage user identity contact with cloud server in the subsequent steps. Similarly, if attacker attempt to deceive user, the attack must have a valid message $\mathrm{C}_{3}$. From the paper we know, the message $\mathrm{C}_{3}$ is encrypted by the private key SK. And because of the characteristics of asymmetric key, it is not possible for attacker decrypt the message $C_{3}$. The attack can't acquire the private key from the TPM platform. The signature can not be falsified. In addition, the attackers don't know the secret number "b" of cloud server. At last, the attack would be failed.

- Cloud platform internal attack: Because user register to cloud server for $\mathrm{h}(\mathrm{PW} \oplus \mathrm{n})$ in stead of $\mathrm{PW}$, the cloud platform internal personnel can't know PW directly. And the number of " $\mathrm{n}$ " is not send to cloud server, attacker can't get PW by offline guessing attack from the message $h(P W \oplus n)$. So the scheme can resist this attack.

- Forward secrecy attack: Through eavesdropping the login and verification process between user and cloud server the attacker will get the message of $\left\{I D, C_{1}, C_{2}, C_{3}, K_{U}, K_{S}, L, T_{1}, T_{2}\right\}$. From $\mathrm{C}_{1}, \mathrm{C}_{2}, \mathrm{C}_{3}$ and $\mathrm{L}$ can't get useful information. $\mathrm{K}_{\mathrm{U}}=\mathrm{g}^{\mathrm{a}}$ $\bmod \mathrm{P}, \mathrm{K}_{\mathrm{S}}=\mathrm{g}^{\mathrm{b}} \bmod \mathrm{P}, \mathrm{K}_{\mathrm{US}}=\left(\mathrm{g}^{\mathrm{a}}\right)^{\mathrm{b}} \bmod \mathrm{P}$. The attacker can't obtain the session key $\mathrm{K}_{\mathrm{US}}$ form $\mathrm{K}_{\mathrm{U}}$ and $\mathrm{K}_{\mathrm{s}}$. The above information are noting with password, Even get user's password, the attacker still can't obtain user and server previous sharing key.

\section{B. Performance Analysis}

The computing time of this scheme meet the requirements of cloud computing environment. All kinds of computing time symbols defined as shown in table II . Analysis shows that the scheme in the paper computing time are $\mathrm{T}=13 \mathrm{~T}_{\mathrm{h}}+12 \mathrm{~T}_{\text {xor }}+4 \mathrm{~T}_{\text {exp }}+1 \mathrm{~T}_{\mathrm{sig}}+1 \mathrm{~T}_{\mathrm{pk}}+2 \mathrm{~T}_{\mathrm{PCR}}+1 \mathrm{~T}_{\text {log. Among }}$ them, the registered stage are $2 \mathrm{~T}_{\mathrm{h}}+2 \mathrm{~T}_{\mathrm{xor}}$, the login stage are $9 \mathrm{~T}_{\mathrm{h}}+7 \mathrm{~T}_{\mathrm{xor}}+4 \mathrm{~T}_{\text {exp }}+1 \mathrm{~T}_{\mathrm{sig}}+1 \mathrm{~T}_{\mathrm{pk}}+2 \mathrm{~T}_{\mathrm{PCR}}+1 \mathrm{~T}_{\text {log }}$, the password change stage are $2 \mathrm{~T}_{\mathrm{h}}+3 \mathrm{~T}_{\mathrm{xor}}$. In the stage of validate the credibility of the cloud server platform, the increase time is $2 \mathrm{~T}_{\mathrm{PCR}}+1 \mathrm{~T}_{\text {log. }}$. The validation just need a couple of times hash operation. User can select a certain number randomly from PCR for validation. So in order to provide more security of the scheme the increase time is necessary.

TABLE II. NOTATIONS

\begin{tabular}{cl}
\hline Symbol & \multicolumn{1}{c}{ Definition } \\
\hline $\mathrm{T}_{\mathrm{h}}$ & The time of hash operation \\
$\mathrm{T}_{\mathrm{xor}}$ & The time of XOR operation \\
$\mathrm{T}_{\text {exp }}$ & The time of exponential operation \\
$\mathrm{T}_{\mathrm{sig}}$ & The time of signature operation \\
$\mathrm{T}_{\mathrm{pk}}$ & The time of decryption operation \\
$\mathrm{T}_{\mathrm{PCR}}$ & The time of calculate PCR \\
$\mathrm{T}_{\log }$ & The time of load SML \\
\hline
\end{tabular}

The proposed work has been implemented using Microsoft Visual C++6.0 and SQL Server 2000 as the backed on Windows Platform. The Hardware and Software Configurations used as follows. The hardware configuration used in this implementation work are: The Intel(R) Core(TM) 2 E7500 processor with the operating frequency $2.93 \mathrm{GHz}$, the Main Memory used is $1.98 \mathrm{~GB}$ RAM, and the 300GB 
TABLE III. PROCESSING TIME FOR VARIOUS OPERATIONS

\begin{tabular}{ccccccccccc}
\hline & 1 & 2 & 3 & 4 & 5 & 6 & 7 & 8 & Average \\
\hline XOR & 1233 & 1189 & 955 & 890 & 8561 & 7516 & 9455 & 8466 & 4783.125 \\
Exponentiation & 455 & 852 & 873 & 4582 & 4686 & 14859 & 45646 & 13774 & 10715.875 \\
Hash & 824553 & 1454260 & 127645 & 57842 & 65542 & 65466 & --- & --- & 432551.333 \\
Encryption & 1256731 & 5614958 & 874214 & 3558642 & --- & --- & --- & --- & 2826136.25 \\
Decryption & 4245666 & 9427670 & 3595233 & 158579 & --- & --- & --- & --- & 4356787 \\
\hline
\end{tabular}

TABLE IV. OPERATIONS AND PROCESSING TIME FOR VARIOUS SCHEMES

\begin{tabular}{|c|c|c|c|c|c|}
\hline & JUANG scheme $^{[9]}$ & FAN scheme $^{[10]}$ & SANTHOSH scheme ${ }^{[11]}$ & YANG scheme $^{[7]}$ & The proposed scheme \\
\hline XOR computations & 1 & 3 & 12 & 12 & 9 \\
\hline Exponentiation & 4 & 1 & --- & 15 & 7 \\
\hline Hash computations & 5 & 4 & 6 & 4 & 4 \\
\hline Encryption & 3 & 3 & 2 & 1 & 1 \\
\hline Decryption & 3 & 4 & 2 & 1 & 1 \\
\hline Total processing time & 23759143.04 & 27660827.332 & 17018551.998 & 9131264.207 & 9031187.832 \\
\hline
\end{tabular}

Hard Disk Drive. The software configurations used are: 32Bit Microsoft Windows XP Operating System, the C++ is used as Front End, and the SQL Server 2000 is used as backend. The length of all kinds of operation data is less than 256 bit. The processing time of each operation in login stage is shown in tableIII.

TableIV shows the operations and the total processing time(in nanoseconds) for different smart card authentication schemes [7,9-11] along with the proposed scheme based on the hardware and software configurations used in the implementation of this authentication scheme.

Analysis shows that the proposed scheme consumes less processing time, when compared with the other mentioned authentication schemes. And the scheme has realized more security goals. Therefore, the proposed authentication scheme performs better than the other compared and discussed smart card authentication schemes.

\section{CONCLUSION}

This article discussed a new smart card mutual authentication scheme based on the trusted cloud computing platform. The scheme completes the authentication of both sides in cloud computing, generates the session key according consulting, at the same time, verifies the credibility of cloud service platform. This mutual authentication scheme has been implemented. The security analysis shows that the proposed scheme provides more security features and overcomes all the discussed security attack or threats because of the feature of TPM. The performance analysis shows that the proposed scheme takes much less computation time than the other discussed smart card authentication schemes. Due to these features, the proposed scheme is a well-secured scheme for smart card in cloud computing environment.

\section{ACKNOWLEDGMENT}

This work was supported by National Science and Technology Support Program (2013BAK07B04).

\section{REFERENCES}

[1] Kang Chen, Weiming Zheng. "Cloud Computing: System Instances and Current Research,” Journal of Software, vol. 20, pp. 1337-1348, 2009.

[2] Junzhou Luo, Jiahui Jin, Aibo Song, et al. "Cloud Computing: Architecture and Key Technologies,” Journal on Communications, vol. 32, pp. 3-21, 2011.

[3] Dengguo Feng, Min Zhang, Yang Zhang, et al. "Study on Cloud Computing Security,” Journal of Software, vol.22, pp. 71-83, 2011.

[4] Dengguo Feng, Yu Qin, Dan Wang, et al. "Research on Trusted Computing Technology,” Journal of Computer Research and Development, vol. 48, pp. 1332-1349, 2011.

[5] "Towards Trusted Cloud Computing" http://www.usenix.org/events/hotcloud09/tech/full_papers/santos.pdf. 2009.

[6] Xian Xu, Yu Long, Xian Ping Mao. "Research on TPM based Strong ID Authentication Protocol,” Computer Engineering, vol. 38, pp. 2327, 2012.

[7] Li Yang, Jianfeng Ma. "Trusted Mutual Authentication Scheme with Smart Cards and Passwords," Journal of University of Electronic Science and Technology of China, vol. 40, pp. 128-133, 2011.

[8] Tien Ho Chen, Han Cheng Hsiang, Wei Kuan Shih. "Security Enhancement on An Improvement on Two Remote User Authentication Schemes Using Smart Cards," Future Generation Computer Systems, vol. 27, pp. 377-380, 2011.

[9] Wen Shenq Juang. "Efficient Password Authenticated Key Agreement Using Smart Card,” Computer and Security, vol. 23, pp. 167-173, 2004.

[10] Chun I Fan, Yihui Lin and Ruei Hau Hsu. "Remote Password Authentication Scheme with Smart Cards and Biometrics," Proceedings of the IEEE Global Telecommunications Conference (GLOBECOM'06). San Francisco, CA, USA: IEEE Press, 2006, pp. 1-5.

[11] S. Santhosh Baboo, K.Gokulraj. “An Enhanced Dynamic Mutual Authentication Scheme for Smart Card Based Networks," Computer Network and Information Security, vol. 4, pp. 30-38, 2012. 